\title{
Frontières
}

\section{Développement d'une approche intégrée de soins curatifs et palliatifs}

\section{Aux personnes atteintes d'insuffisance cardiaque sévère en centre hospitalier}

\section{Sylvie Décarie, Sylvie Cossette, Réjeanne Dubeau et Sonia Heppell}

Volume 20, numéro 1, automne 2007

La « bonne mort»

URI : https://id.erudit.org/iderudit/017949ar

DOI : https://doi.org/10.7202/017949ar

Aller au sommaire du numéro

Éditeur(s)

Université du Québec à Montréal

ISSN

1180-3479 (imprimé)

1916-0976 (numérique)

Découvrir la revue

Citer cet article

Décarie, S., Cossette, S., Dubeau, R. \& Heppell, S. (2007). Développement d'une approche intégrée de soins curatifs et palliatifs : aux personnes atteintes d'insuffisance cardiaque sévère en centre hospitalier. Frontières, 20(1), 62-68. https://doi.org/10.7202/017949ar
Résumé de l'article

L'insuffisance cardiaque (IC) est une maladie chronique complexe, touchant plus de 300000 Canadiens, qui évolue vers une phase terminale. Le taux de mortalité s'élève à $50 \%$ dans la première année suivant le diagnostic d'IC de stade IV. L'émergence des soins palliatifs a permis de centrer les soins sur la qualité de vie, le soulagement de la douleur et des symptômes en fin de vie. Plusieurs études relatent que les personnes atteintes d'IC à un stade avancé présentent des symptômes similaires aux personnes atteintes de cancer. Cependant, les personnes qui bénéficient des soins palliatifs sont surtout celles atteintes de cancer. Un centre de cardiologie tertiaire de la région de Montréal a implanté une approche intégrée de soins curatifs et palliatifs pour la clientèle IC. La trajectoire particulière de l'IC rend complexe et essentiel le développement d'une approche intégrée de soins curatifs et palliatifs pour maximiser la qualité de vie des personnes atteintes d'IC en fin de vie.
Ce document est protégé par la loi sur le droit d'auteur. L'utilisation des services d’Érudit (y compris la reproduction) est assujettie à sa politique d'utilisation que vous pouvez consulter en ligne.

https://apropos.erudit.org/fr/usagers/politique-dutilisation/ 


\section{Résumé}

L'insuffisance cardiaque (IC) est une maladie chronique complexe, touchant plus de 300000 Canadiens, qui évolue vers une phase terminale. Le taux de mortalité s'élève à $50 \%$ dans la première année suivant le diagnostic d'IC de stade IV. L'émergence des soins palliatifs a permis de centrer les soins sur la qualité de vie, le soulagement de la douleur et des symptômes en fin de vie. Plusieurs études relatent que les personnes atteintes d'IC à un stade avancé présentent des symptômes similaires aux personnes atteintes de cancer. Cependant, les personnes qui bénéficient des soins palliatifs sont surtout celles atteintes de cancer. Un centre de cardiologie tertiaire de la région de Montréal a implanté une approche intégrée de soins curatifs et palliatifs pour la clientèle IC. La trajectoire particulière de I'IC rend complexe et essentiel le développement d'une approche intégrée de soins curatifs et palliatifs pour maximiser la qualité de vie des personnes atteintes d'IC en fin de vie.

Mots clés: insuffisance cardiaque intégration des soins - soins palliatifs programme de soins hospitaliers.

\section{Abstract}

Heart failure (HF) is a chronic and incurable disease evolving toward a terminal phase. More than 300000 Canadians suffered from HF. Patients with a severe form of HF (phase IV, according to the New York Heart Association) have a one year survival of $50 \%$. Palliative care has been extensively developed over the last years for the benefit of the cancer patient, these cares emphasizes a biopsychosocial approach that includes quality of life, pain control and symptoms relief at the end of life. Unfortunately, palliative care is rather poorly offered to the people suffering from a non malignant condition. Many authors stated that the symptoms of HF failure patients are quite similar to those observed for palliative cancer patients. The Montreal Heart Institute (MHI) has integrated a palliative care approach according to the Quebec policy on palliative care. This palliative care approach contributes to the quality of care and promotes the patients and clinicians satisfaction.

Keywords: heart failure integrated care - palliative care hospital care program.

\section{DÉVELOPPEMENT D'UNE APPROCHE INTÉGRÉE DE SOINS CURATIFS ET PALLIATIFS Aux personnes atteintes d'insuffisance cardiaque sévère en centre hospitalier}

\author{
Sylvie Décarie, M.Sc., \\ infirmière et conseillère en soins spécialisés, \\ CLSC-CSSS Lucille-Teasdale. \\ Sylvie Cossette, Ph.D., \\ infirmière, professeure agrégée, Faculté des sciences \\ infirmières, Université de Montréal, et chercheuse, \\ Institut de cardiologie de Montréal. \\ Réjeanne Dubeau, M.Sc., \\ infirmière et conseillère en soins spécialisés, \\ Institut de cardiologie de Montréal. \\ Sonia Heppell, M.Sc., \\ infirmière, IPSC, Institut de cardiologie de Montréal.
}

Notre expérience professionnelle acquise en centre hospitalier de soins aigus et en santé communautaire a permis d'observer les difficultés auxquelles sont confrontées les personnes souffrant d'insuffisance cardiaque (IC) sévère. Ces personnes nous ont souvent confié avoir l'impression de «tomber entre deux chaises » en raison du stade avancé de leur maladie. La coexistence de soins curatifs et des soins assurant le confort génère une ambigüité quant à l'im- portance de chacun de ces soins permettant d'assurer une qualité de vie optimale tout au long de l'évolution de la maladie. En général, ces personnes malades polysymptomatiques vivent un niveau élevé de détresse et d'inconfort et reçoivent peu d'accompagnement puisque l'aspect curatif prédomine dans les modèles de soins aigus. L'absence d'approche palliative pour la clientèle IC sévère hospitalisée constitue une problématique clinique sur laquelle il est essentiel de réfléchir et d'agir.

\section{L'INSUFFISANCE CARDIAQUE}

L'IC se définit comme étant l'incapacité du cœur à répondre aux besoins métaboliques de l'organisme. Il s'agit d'une maladie chronique à caractère complexe touchant plus de 300000 Canadiens et pour laquelle on estime l'incidence à plus de 40000 cas par année (Ducharme, 1999). L'évolution de l'IC s'accompagne de symptômes qui sont regroupés sous quatre classes fonctionnelles de sévérité (I à IV) d'après l'Association new-yorkaise des maladies 
du cœur (Bowling, 1995). L'individu souffrant d'IC sévère de stade III rapporte de la fatigue, de la dyspnée, des palpitations, ou de l'angine lors d'activités modérées tandis qu'au stade IV, ces symptômes sont présents même au repos. L'intensité des traitements à préconiser par les cliniciens se posera de plus en plus avec la progression de la maladie.

À Montréal, le taux annuel moyen d'hospitalisation de cette clientèle a augmenté de 26\% depuis 1990 (Régie régionale de la santé et des services sociaux de Montréal-Centre, 1999). En fait, la mortalité des personnes atteintes d'IC est de 40 à $50 \%$ dans la première année suivant le diagnostic d'IC de stade III ou de stade IV (McMurray et Stewart, 2000; Pantilat et Steimle, 2004). L'accroissement important de cette clientèle en milieu hospitalier favorisera le développement d'approches novatrices intégrées de soins curatifs et de soins palliatifs au cours des prochaines années. À l'heure actuelle, il y a des orientations ministérielles pertinentes concernant les soins palliatifs (ministère de la Santé et des Services sociaux, MSSS, 2004). Cependant, peu de milieux hospitaliers ultraspécialisés ont opérationnalisé l'approche intégrée de soins curatifs et palliatifs aux patients souffrant de maladies cardiovasculaires avancées.

Les personnes souffrants d'IC, particulièrement à un stade avancé de la maladie, éprouvent plusieurs symptômes généraux (tels que la fatigue, la douleur globale, de la faiblesse et de l'insomnie), cardiaques (dyspnée, palpitations, angine) et gastro-intestinaux (constipation, nausées et vomissements) relatifs au phénomène de l'hypoperfusion de plusieurs organes vitaux (Deaton et Grady, 2004; Kutner Kassner et Nowels, 2001; McCarthy, Lay et Addington-Hall, 1996; McCarthy, Addington-Hall et Lay, 1997). Une étude très importante menée par Levenson, McCarthy, Lynn, Davis et Phillips (2000) auprès des personnes atteintes d'IC sévère relate que $35 \%$ présentaient des douleurs sévères et $43 \%$ souffraient de dyspnée En toute fin de vie, $43 \%$ éprouvaient des douleurs sévères et $65 \%$ de la dyspnée. Ces patients souffrent aussi de symptômes psychologiques tels des symptômes dépressifs, de l'anxiété et présentent des besoins sociaux et spirituels qui sont peu considérés (Murray et al., 2002). L'évolution de la maladie s'accompagne d'une augmentation significative des symptômes qui entraînent souvent des réadmissions à l'hôpital, ce qui génère des coûts importants mais est surtout vécu comme un événement non souhaité par le patient et sa famille. En outre, les résultats de certaines études (Anderson et al., 2001; Horne et Payne,
2004 ; Lynn, Teno et Russell, 1997) ont révélé que les symptômes et difficultés éprouvés par les personnes souffrant d'IC sévères étaient similaires à ceux des personnes atteintes de cancer en phase terminale, mais variaient en intensité selon la pathologie sous-jacente. Une étude prospective (Anderson et al., 2001) menée auprès de patients atteints d'IC en phase terminale a permis d'observer que plus de la moitié de ces patients n'avaient pas obtenu d'amélioration en regard des problèmes physiques et autant avaient reçu peu ou pas d'interventions en regard de leurs difficultés psychosociales.

L'évolution de l'IC peut être ralentie jusqu'à un certain point par l'application de traitements pharmacologiques (médications tels les diurétiques, les inhibiteurs de l'enzyme de conversion de l'angiotensine (IECA), les antagonistes des récepteurs de l'angiotensine (ARA), les Bêta-bloqueurs, les vasodilatateurs, les inotropes positifs, les antiarythmiques) et non pharmacologiques (diète hyposodée, restriction liquidienne, activités physiques) qui permettent d'améliorer la condition clinique des patients (Doyon, 2002; Hendrick, 2001 ; Remme et Swedberg, 2001). Il n'en demeure pas moins que certains facteurs tels que la difficulté d'être assidu au traitement recommandé, la présence de co-morbidités et le caractère évolutif de la maladie peuvent entraîner d'autres réadmissions (Moser et Worster, 2000). Ces réadmissions impliquant des transitions entre l'hôpital et le domicile suscitent de la peur et de la détresse chez la personne atteinte d'IC, ce qui peut contribuer à accentuer aussi le fardeau des proches soignants (Murray et al., 2002). Toutes ces difficultés à composer avec les exigences de cette maladie ont pour effet d'altérer la qualité de vie de l'individu atteint d'IC de même que celle de sa famille (Juenger, Schellberg et Kraemer, 2002).

Il existe une difficulté incontournable dans l'évaluation de la trajectoire de la maladie chez les patients atteints d'IC sévère. Contrairement aux patients cancéreux, qui perdent progressivement leurs capacités à mesure qu'évolue la maladie, l'IC est ponctuée de plateaux plus ou moins hauts et longs, soit des périodes de stabilité et même d'amélioration de l'état fonctionnel, intercalés de périodes fréquentes et répétées d'exacerbations (Goodlin et al., 2004). Les éléments pronostiques habituels tels qu'une altération importante de la fonction rénale et la perte de poids ont une valeur prédictive très limitée et encore une fois sont variables d'un patient atteint d'IC à l'autre. À l'heure actuelle, il s'avère donc très difficile d'identifier clairement la trajectoire de la maladie chez les patients atteints d'IC sévère et donc d'adopter des interventions appropriées tout au long de la maladie. Par ailleurs, il ne fait aucun doute que l'IC évolue vers une phase terminale pour laquelle on compte près de $50 \%$ de mort subite (Pantilat et Steimle, 2004).

\section{LES BESOINS DES PERSONNES} ATTEINTES D'IC EN FIN DE VIE

Des études révèlent que seulement 18\% à $25 \%$ des patients IC hospitalisés pour une détérioration importante de leur condition se souviennent avoir eu des discussions avec un professionnel, par exemple quant à leurs choix face à la réanimation cardiorespiratoire (Krumholz et al., 1998; McCarthy, Lay et Addington-Hall, 1996; McCarthy, Addington-Hall et Lay, 1997). En comparaison avec des patients cancéreux en fin de vie, les patients atteints d'IC sévère rapportent avoir reçu moins d'informations sur leur état de santé, démontrent une compréhension moindre de la maladie et du pronostic et sont moins impliqués dans la prise de décision (Murray et al., 2002). Pourtant, la majorité $(80 \%)$ des patients en fin de vie souhaitent avoir une discussion ouverte avec l'équipe de soins à propos de l'évolution de leur maladie (Butow, Dowsett, Hagerty et Tattersall, 2002 ; Finucane, 2002). Les préoccupations des personnes atteintes d'IC avancée rejoignent celles des autres malades affligés d'une maladie chronique évolutive ou d'un cancer. L'ensemble de ces malades désirent avoir un meilleur contrôle de la douleur et des symptômes, éviter la prolongation non désirée du processus du mourir, participer aux décisions concernant les soins, renforcer les relations avec les proches et enfin finaliser certaines tâches (Singer, Martin et Kelner, 1999; Steinhauser et al., 2001).

D'autre part, contrairement aux patients cancéreux en toute fin de vie pour qui un traitement curatif n'est plus indiqué, certains traitements plus agressifs utilisés lors des nombreuses périodes de décompensation de la maladie cardiaque sont appropriés jusqu'à la toute fin de vie chez la clientèle atteinte d'IC sévère, particulièrement si ces traitements permettent le soulagement des symptômes physiques de même que l'amélioration de la qualité de vie au plan psychologique, interpersonnel et spirituel (Goodlin et al., 2004). Il est possible que la trajectoire labile de l'IC et l'absence de reconnaissance que l'IC sévère est une maladie terminale entraînent des problèmes de communication entre les professionnels, les patients et leur famille face aux étapes à venir et aux possibilités de traitement. Les guides de pratique clinique (guidelines) en IC actuellement disponibles et élaborés entre autres par 
l'American Heart Association (AHA) et l'American College of Cardiology (ACC) concernent les soins en phase aiguë de la maladie et excluent de façon générale les patients les plus malades ou dont le décès est plus ou moins prévisible à court terme. Ces études visent l'évaluation de la médication ou de certaines approches thérapeutiques sur la mortalité et la morbidité de la clientèle IC, mais ne considèrent pas spécifiquement l'amélioration de la qualité de vie et le confort des insuffisants cardiaques sévères.

En résumé, il ressort que les besoins de la plupart des personnes atteintes d'IC sévère et de leurs proches ne sont pas suffisamment considérés sur les plans physique, psychosocial et spirituel en regard d'une approche intégrée de soins à la fois curatifs et palliatifs. Pour la grande majorité de la clientèle IC, une approche intégrée de soins curatifs et palliatifs pourrait ajouter aux modèles existants dans le but d'identifier et de répondre de façon plus spécifique et globale aux besoins.

\section{LES SOINS PALLIATIFS}

La mort est souvent repoussée, particulièrement dans les milieux hospitaliers qui visent à combattre la maladie et à vaincre la mort même si ces milieux demeurent le lieu le plus important où les patients décèdent des suites de maladies. En effet, la majorité des Québécois décèdent dans des centres hospitaliers même si la plupart d'entre eux préféreraient mourir à domicile (MSSS, 1998). Certaines estimations indiquent que $5 \%$ des Canadiens ont accès à des soins palliatifs, bien que près de $70 \%$ des personnes décèdent d'une maladie susceptible de requérir des soins palliatifs en fin de vie. Ainsi, une majorité de Canadiens atteints de cancer ou d'autres maladies non malignes incurables ne reçoivent pas de services et de soins palliatifs ou, s'ils en reçoivent, ceux-ci sont jugés très insuffisants. À cet égard, le rapport de Lambert et Lecomte (2000) est éloquent: les soins palliatifs sont offerts de façon inégale au Québec et sont malheureusement marginalisés dans le continuum de soins et de services de santé.

$\mathrm{Au}$ cours des dernières années, les soins prodigués aux personnes atteintes de maladies incurables se sont largement améliorés en ce qui a trait aux traitements des symptômes de nature physique (douleur, nausée, vomissement, etc.), psychosociale (anxiété, isolement, etc.) et spirituelle (recherche du sens de la vie, etc.) (Davies et Curtis, 2000). En effet, l'émergence des soins palliatifs a permis de centrer les soins sur la qualité de vie, le contrôle de la douleur et le soulagement des symptômes en fin de vie. De façon plus large, la prestation des soins palliatifs englobe les principes éthiques tels le respect de l'autonomie, la non-malfaisance, la bienfaisance et enfin celui de la justice (Beauchamp et Childress, 1994).

La prestation de ces soins réduit la durée d'hospitalisation dans les unités de soins aigus et permet un retour à domicile pour plusieurs de ces patients (Hearn et Higginson, 1998). De façon générale, les soins palliatifs s'adressent à des personnes de tous âges souffrant de traumatismes graves, de maladies incurables ou de maladies liées au vieillissement (MSSS, 2004). Cependant, les personnes pouvant bénéficier de soins palliatifs sont majoritairement celles atteintes de cancer, ce qui soulève des questions quant à l'accessibilité pour d'autres clientèles souffrant de maladies terminales. Il est estimé que 5 à $10 \%$ de l'ensemble des personnes atteintes de maladies terminales reçoivent ce type de soins au Québec. Ceux n’y ayant pas accès décèdent avec des douleurs peu contrôlées, de l'inconfort et des besoins psychosociaux plus ou moins comblés (Lambert et Lecomte, 2000).

Par ailleurs, il faut souligner que pour plusieurs cliniciens les soins palliatifs sont considérés comme étant des soins destinés aux patients en toute fin de vie ne répondant plus aux traitements curatifs. Cependant, de plus en plus de cliniciens introduisent le concept de soins palliatifs aussitôt qu'un individu reçoit un diagnostic de maladie pour laquelle il n'existe pas de guérison et à issue éventuellement fatale. L'Association canadienne des soins palliatifs (ACSP) souhaite que «les soins palliatifs ne soient plus considérés comme des soins destinés aux mourants, mais bien des soins ayant pour but de soulager la

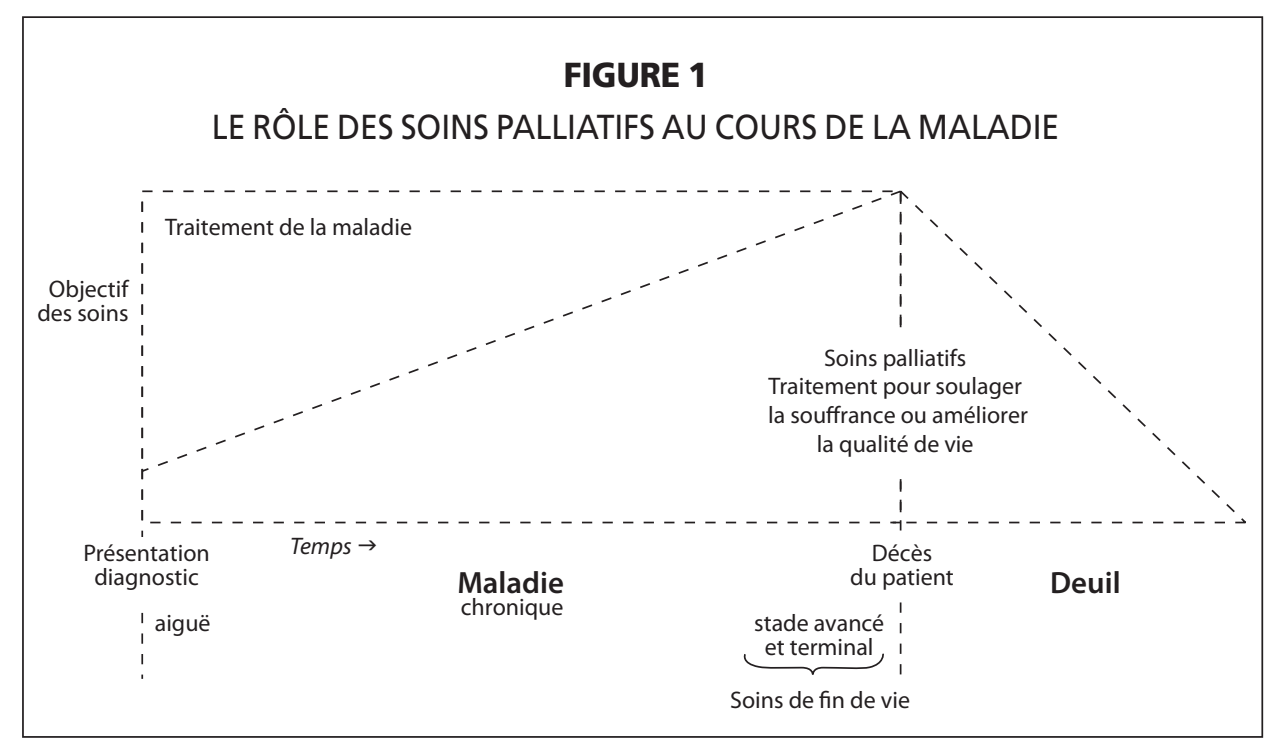

Source: Ferris et al., «Modèle de guide des soins palliatifs : fondés sur les principes et les normes de pratiques nationaux», ACSP, 2002. souffrance et d'améliorer la qualité de vie pendant toute l'expérience de la maladie et du deuil » (Ferris et al., 2002, p. vi). Dans le même ordre d'idées, le MSSS (2004) a émis une politique sur les soins palliatifs de manière à favoriser l'accessibilité, la continuité et la qualité des soins palliatifs dans la même perspective que l'ACSP et l'Organisation mondiale de la santé (OMS), soit avec une approche holistique et humaniste (voir figure 1). Il faut reconnaître que les soins palliatifs au Québec varient encore d'une institution à une autre selon la disponibilité des ressources et souffrent d'un problème de coordination dans le continuum de soins.

Les soins palliatifs aident les patients à exprimer les pertes vécues quant aux relations avec les proches et renforcent l'utilisation des forces du patient pour composer avec la maladie (Faull et Woof, 2002). La prestation des soins palliatifs par une équipe interdisciplinaire a permis de relever une amélioration significative du soulagement de la douleur et des symptômes de patients souffrant de cancer avancé (Higginson et al., 2002; Ellershaw et Wilkinson, 2003; Lynch et Chamberlain, 1998). Les soins palliatifs améliorent la satisfaction des familles en regard des soins offerts aux patients tout en permettant de réduire l'intensité des interventions à visée curative (Campbell, 1996). On observe aussi une amélioration de la qualité de vie des patients hospitalisés en soins palliatifs comparativement à ceux d'un groupe contrôle (Viney, Walker, Robertson, Lilley et Ewan, 1994). Par ailleurs, Meier (2002) révèle que les personnes atteintes d'un cancer ayant reçu des soins palliatifs rapportent un niveau élevé de satisfaction pour le contrôle de 
la douleur (95\%), des symptômes (92\%), pour l'amélioration de la qualité de vie $(84 \%)$ et pour le soutien à la famille $(84 \%)$. L'accès aux soins palliatifs a permis de réduire la durée d'hospitalisation de personnes cancéreuses en phase terminale en raison de l'accessibilité à des soins palliatifs à domicile ou en établissements spécialisés, en Alberta (Fainsiger et al., 2003). Lynn, Schuster et Kabcenell (2000) relatent une diminution d'hospitalisation de 40 jours par année par patient à la suite de l'implantation de soins palliatifs auprès de personnes atteintes de maladies cardiaques terminales aux États-Unis. Des études menées en Angleterre au cours des années 1990 mentionnent que les soins palliatifs permettent de réduire les coûts $\mathrm{du}$ fait que les personnes malades sont hospitalisées moins longtemps et que l'intensité des soins est moindre (AddingtonHall et al., 1992; Hughes et al., 1992). Il demeure qu'il n'existe pas d'études qui font état de la diminution des coûts liée à l'implantation des soins palliatifs au Québec.
Les effets bénéfiques issus de la prestation de soins palliatifs auprès de patients atteints de maladies malignes suggèrent que ces soins pourraient être également offerts à d'autres clientèles atteintes de maladies non malignes (O'Brien, Welsh et Dunn, 1998). Les personnes atteintes d'IC sévère présentent une panoplie de symptômes et de besoins comparables à ceux des personnes souffrant de cancer avancé, mais ne trouvent pas toujours de réponses satisfaisantes dans les milieux cliniques. D'autre part, la trajectoire de l'IC est peu prévisible dans le temps, ce qui rend difficile la planification des soins palliatifs dans ce contexte. En fait, les soins palliatifs reposent sur le travail d'une équipe interdisciplinaire ayant ciblé des objectifs communs visant à rehausser la qualité de vie et le confort de la clientèle. Les intervenants de l'équipe de soins en milieu aigu reconnaissent ne pas disposer de toutes les connaissances et habiletés pour offrir les soins de fin de vie requis par la clientèle IC sévère hospitalisée.
Les professionnels œuvrant en soins aigus ont généralement reçu une formation centrée sur des interventions à visée essentiellement curative. Les soins prodigués aux personnes atteintes d'IC sévère sont perçus comme étant complexes et nécessitant une approche de soins globale auprès du patient et de sa famille pour laquelle les soignants se sentent peu outillés. Wotton, Borbasi et Redden (2005) ont analysé la pratique clinique d'un groupe d'infirmières travaillant en soins aigus. Ces auteurs rapportent que les infirmières sont confrontées à plusieurs enjeux en ce qui a trait aux difficultés de communication entre patients, infirmières, médecins et autres professionnels de l'équipe de soins, au caractère intense et expéditif des soins aigus, au manque de soutien clinique et à l'absence de structure organisationnelle pour offrir les soins palliatifs à la clientèle. Ces infirmières estiment que ces patients devraient pouvoir obtenir un meilleur soulagement de la douleur et des symptômes. Cependant, cela doit se faire dans



Source: Ferris et al., Modèle de guide des soins palliatifs: fondé sur les principes et les normes de pratique nationaux, ACSP, 2002. 


\section{ENCADRÉ 1}

\section{HISTOIRE VÉCUE DE L'APPLICATION DE L'APPROCHE INTÉGRÉE DE SOINS PALLIATIFS ET CURATIFS AUPRĖS D'UNE PERSONNE ATTEINTE D'IC SÉVÈRE}

Madame X a été hospitalisée à plusieurs reprises pour défaillance cardiaque au cours des derniers mois. Elle demeure avec son conjoint depuis plusieurs années et entretient avec lui une excellente relation. Elle est aussi très bien entourée et soutenue par ses frères et sœurs. L'équipe de soins palliatifs a été consultée lors de sa dernière hospitalisation et a examiné les quatre sphères d'évaluation et d'interventions, soit physique, psychologique, interpersonnelle et spirituelle.

L'évaluation a d'abord révélé une souffrance physique importante en raison de l'œdème (enflure) aux jambes et à l'abdomen; de plus, madame X est affligée d'une condition arthritique sous-jacente qui la rendait extrêmement inconfortable et qui nuit à sa mobilité. Le pharmacien et le médecin de l'équipe ont débuté et ajusté les analgésiques de façon à permettre à madame d'être confortable et de retrouver une certaine forme de bien-être physique. De plus, l'inquiétude quant à l'évolution de sa condition de santé, associée à de la dyspnée paroxystique nocturne (sensation d'étouffement la nuit) perturbait son sommeil. Des ajustements médicamenteux ont également été faits à cet égard, ce qui a permis à madame $\mathrm{X}$ de retrouver un sommeil réparateur. Les infirmières de l'équipe ont eu pour rôle principal d'offrir le soutien psychologique (communication thérapeutique avec le couple). Madame X a toujours montré une certaine sérénité quant à la détérioration constante de son état de santé et un réalisme parfois surprenant. Son conjoint, par contre, était triste et anxieux. Il démontrait un grand désarroi et une certaine forme de déni quant à la détérioration rapide et récente de sa conjointe. Il a été important d'aider le couple à cheminer dans l'évolution de la maladie. Après 4 semaines d'hospitalisation et l'atteinte d'un meilleur état de bien-être physique, des démarches ont été entreprises par le travailleur social et l'infirmière de liaison de l'équipe afin de permettre à madame $\mathrm{X}$ de retourner à domicile puisque c'était là son souhait. En effet, madame voulait aller passer quelques semaines chez elle afin de revoir son potager et faire quelques pas dans son jardin... une dernière fois. Ce retour à domicile représentait un défi de taille pour l'équipe. En effet, la patiente nécessitait l'administration de plusieurs doses importantes de diurétique intraveineux à chaque jour, ce qui actuellement ne peut être assumé par les services restreints de la plupart des ressources hors hospitalières (soins à domicile des CLSC). De même, des équipements (lit électrique, oxygène, etc.) devaient être installés à domicile afin de permettre à madame $\mathrm{X}$ de rentrer chez elle. Avant le départ de madame, l'équipe de soins palliatifs a communiqué avec le centre de soins palliatifs de la région, afin de s'assurer qu'au moment opportun, les ressources hospitalières en soins palliatifs de sa région seraient au courant de sa condition et donc disponibles à la recevoir. La mise en place de ce corridor de services, la collaboration de toute l'équipe de soins palliatifs avec la famille de madame et avec l'équipe des soins à domicile de sa région ont permis de répondre aux souhaits de fin de vie de madame et de son conjoint. Madame X a vécu 3 semaines chez elle, entouré de sa famille et des êtres qui lui étaient chers. Elle a ensuite été admise dans un centre de soins palliatifs où elle est décédée un mois plus tard.

Madame X, avant son décès, et son conjoint nous ont exprimé leur reconnaissance quant au soutien reçu et la disponibilité, l'écoute et la présence des professionnels de l'équipe des soins palliatifs. Ils ont indiqué que la considération de l'ensemble de leurs besoins a permis de diminuer les souffrances physiques de madame, mais également de réduire leur souffrance psychologique en tant que couple. Notre expérience, de plus d'une année maintenant, nous permet de réaliser la valeur ajoutée que représente une équipe de soins palliatifs dans le milieu hospitalier tertiaire cardiologique qu'est l'ICM.

Un retour sur les expériences des dernières années nous amène à constater en tant qu'équipe qu'il reste beaucoup à faire et qu'il existe des difficultés importantes dans la mise en place d'une telle approche, qu'il faudra tenter de surmonter. Par exemple, il arrive que le personnel soignant fasse appel à l'équipe de soins palliatifs tardivement dans l'épisode de soins, quelquefois dans les quelques jours ou quelques heures précédant le décès. Dans l'exemple ci-dessus, madame $\mathrm{X}$ aurait pu bénéficier d'une référence à l'équipe de soins palliatifs lors de ses hospitalisations précédentes, ce qui aurait permis le soulagement de ses souffrances plus rapidement. De même, il aurait été possible de planifier plus judicieusement le retour à domicile avec les partenaires concernés et d'éviter les nombreuses réadmissions à l'hôpital qui représentaient un irritant majeur pour le couple. Également, nous avons constaté que les services de soins à domicile disposent de peu de ressources pour favoriser le maintien à domicile des personnes souffrant d'insuffisance cardiaque en phase de fin de vie. Ces services ont peu d'expériences en ce qui concerne les soins de fin de vie et du «mourir à domicile» de cette clientèle. Cette réalité permet difficilement de répondre aux souhaits de fin de vie de plusieurs patients atteints d'IC sévère. un contexte où de nombreuses demandes sont faites auprès des équipes professionnelles qui n'ont pas toujours l'expertise en matière de soins palliatifs (Hanratty et al., 2002). Ainsi, l'intégration de l'approche palliative aux soins génériques de l'IC n'est pas toujours évidente en raison du manque de connaissances de l'ensemble des professionnels sur les soins palliatifs. Les personnes atteintes d'IC sévère présentent une panoplie de symptômes et de besoins comparables à ceux des personnes souffrant de cancer avancé, mais ne trouvent pas toujours de réponses satisfaisantes à leurs besoins dans les milieux cliniques. Les écrits suggèrent que l'intégration des soins palliatifs aux soins courants de personnes atteintes de maladies chroniques telle l'IC permettrait d'améliorer le confort et la qualité de vie de ces patients et de leurs proches.

\section{PROPOSITION D'UNE APPROCHE INTÉGRÉE DE SOINS CURATIFS ET PALLIATIFS}

La présente réflexion a été effectuée en se basant sur le cadre de référence proposé par Green et Kreuter (1999) qui a permis d'analyser les besoins des professionnels de la santé dont les infirmières œuvrant dans un milieu cardiologique tertiaire soit l'Institut de cardiologie de Montréal (ICM) et ceux de la clientèle IC. Dans ce contexte clinique, les travaux de Décarie (2005) ont permis de constituer une équipe interdisciplinaire en réponse à l'intérêt d'intégrer une approche de soins en fin de vie auprès de la clientèle IC sévère de la part des infirmières, des professionnels de l'équipe soignante et des directions de l'ICM. De plus, cette équipe peut compter sur la présence d'experts en soins cardiologiques dans les équipes médicales, les équipes de recherche, les soins infirmiers et auprès des autres professionnels de l'équipe de soins.

Les réflexions et les constats cliniques partagés par l'ensemble de l'équipe nous ont amenés à proposer un modèle d'évaluation et un nouveau répertoire d'interventions adaptés à cette clientèle (voir figure 2) De plus, une modalité de fonctionnement de l'approche intégrée de soins curatifs et palliatifs destinée à la clientèle IC sévère a été proposée par Décarie (2005). Ainsi, nous constatons que le patient atteint d'une maladie évolutive incurable vit un bouleversement important qui altère tant ses fonctions physiques que sa qualité de vie. La souffrance éprouvée n'est pas seulement physique, mais touche également son intégrité comme être humain, d'où le caractère multidimensionnel de la souffrance. Saunders (1999) a développé le concept de souffrance totale qui est à la 
fois physique, psychologique, interpersonnelle et spirituelle. Les récentes définitions des soins palliatifs englobent désormais tant les maladies malignes que les maladies chroniques évolutives. En effet, ces pathologies montrent des similitudes au niveau de certains symptômes et des besoins à combler pour le patient et sa famille.

Comme illustré à la figure 2 , le modèle mentionne quatre dimensions de la souffrance en lien avec les symptômes et les besoins de la clientèle atteinte d'IC sévère (stades 3 et 4 ). La souffrance physique renvoie à la douleur ainsi qu'à des symptômes chez l'IC sévère concernant la respiration, les tissus et l'appareil gastro-intestinal (Deaton et Grady, 2004). La souffrance psychologique relève des sentiments éprouvés par le patient face aux pertes amenées par la maladie, l'anxiété, les symptômes dépressifs tout comme le manque d'informations sur sa maladie (Murray et al., 2002). La souffrance interpersonnelle concerne l'impact de la maladie sur le système familial tel que le changement des rôles, les difficultés financières, la communication et la dépendance accrue envers les soignants et sa famille (Anderson et al., 2001). Enfin, la souffrance spirituelle intervient lorsque le patient questionne le sens existentiel relatif à sa vie, l'expérience de la maladie et l'appréhension face à la mort (Murray, Kendall, Boyd, Worth et Benton, 2004).

La souffrance est une expérience globale, ce qui requiert la collaboration de l'équipe interdisciplinaire (médecin, infirmière, intervenant social, agent de pastorale, bénévole) et des intervenants collaborateurs qui se réunissent régulièrement pour discuter de chaque situation afin de mieux soutenir l'usager et ses proches et répondre à leurs besoins respectifs. Ces interventions sont centrées sur l'amélioration de la qualité de vie (confort, bienêtre, cheminement du processus de deuil, prise en compte des désirs du patient) et sur son environnement, tant familial que spirituel. La souffrance physique peut être gérée avec la médication relative à l'IC, l'usage d'anxiolytiques et d'opiacés (Albert, Mellar et Young, 2002) de même qu'avec les approches non pharmacologiques telle la musicothérapie (Riley et Blue, 2001) comme l'ont proposé les intervenants du comité interdisciplinaire de l'ICM. Il s'avère important d'offrir une certaine intimité à l'usager et sa famille et de les soutenir par une communication fluide. À cet effet, les patients doivent être informés sur leur maladie, les choix de traitements et le pronostic avec sensibilité et honnêteté (Murray et al., 2002).

Les influences culturelles et religieuses sont d'importance et doivent être prises en considération dans l'approche clinique. Les bénévoles sont des intervenants précieux parce qu'ils peuvent être un lien entre le malade et l'équipe soignante. En ce qui concerne le bien-être spirituel, la présence d'un représentant de la religion d'appartenance du malade est encouragée si ce dernier souhaite qu'il en soit ainsi.

À l'ICM, les travaux issus des rencontres du comité interdisciplinaire ont permis d'émettre des recommandations quant aux besoins de formation afin de favoriser l'implantation de cette approche intégrée auprès de la clientèle atteinte d'IC sévère. En partenariat, les différents professionnels impliqués ont priorisé les besoins de formation liés aux pratiques en soins palliatifs, à la communication interprofessionnelle, à l'interdisciplinarité, aux soins centrés sur la famille, aux concepts de deuil et de perte face à la maladie grave et la fin de vie ainsi que la culture et les rites religieux. Le personnel soignant a reçu cette formation en lien avec les soins palliatifs. Par la suite, une équipe interdisciplinaire «mobile»a été constituée; elle se réunit à chaque semaine depuis 2006 afin de répondre aux besoins de cette clientèle. Une infirmière praticienne spécialisée en cardiologie contribue au développement de cette approche innovatrice en milieu clinique. À ce jour, près de 60 patients-familles ont bénéficié des soins palliatifs à l'ICM (voir l'encadré pour une histoire vécue) et plusieurs revisites à l'urgence ont été évitées. Le succès lié à l'implantation d'une telle approche intégrée repose sur la formation, l'interdisciplinarité et le soutien clinique d'une conseillère en soins spécialisés. Le développement de corridors de services avec les partenaires des différents milieux de soins tels les centres d'hébergements ou les centres locaux de services communautaires (CLSC) permet d'offrir un répertoire de services et de soins concertés pour la clientèle. Dans cette perspective, l'introduction de nouvelles pratiques cliniques a contribué à l'amélioration de la qualité des soins pour la clientèle atteinte d'IC sévère. Les patients-familles et les membres du personnel de l'ICM se disent très satisfaits de l'intégration des soins palliatifs dans le continuum de soins.

Parmi les objectifs que l'équipe de soins palliatifs de l'ICM s'est donnés pour la prochaine année, on retient la sensibilisation et la formation des intervenants du milieu aux bénéfices d'une référence plus précoce à l'équipe de soins palliatifs. De même, nous tenterons d'évaluer les besoins et d'informer les professionnels des services hors hospitaliers à la réalité de fin de vie des patients cardiaques et aux besoins à combler pour leur permettre de vivre à domicile et pour certains d'y décéder entourés de leurs proches. Ceci devrait permettre le développement de services mieux adaptés à la clientèle et la collaboration avec les partenaires du réseau de la santé de façon à favoriser le maintien à domicile lorsque cela est souhaité par le patient et sa famille.

\section{Bibliographie}

ADDINGTON-HALL, J.M., L.D. MacDONALD, H.R. ANDERSON, J. CHAMBERLAIN, P. FREELING, J.M. BLAND et J. RAFFERTY (1992). "Randomised controlled trial of effects of coordinating care for terminally ill cancer patients », British Medical Journal, n 305 , p. 1317-1322.

ALBERT, N., D. MELLAR et J. YOUNG (2002). «Improving the care of patients dying of heart failure », Cleveland Clinic Journal of Medicine, $\mathrm{n}^{\circ}$ 69, p. 321-328

ANDERSON, H., C. WARD, A. EARDLY, S.A. GOMM, M. CONNOLLY, T. COPPINGER, D. CORGIE, J.L. WILLIAMS et J.L. MAKIN (2001). "The concerns of patients under palliative care and a heart failure clinic are not being met», Palliative Medicine, $\mathrm{n}^{\circ}$ 15, p. 279-286.

BEAUCHAMP, T.L. et J.F. CHILDRESS (1994). The Principles of Biomedical Ethics, $4^{\mathrm{e}}$ édition, Oxford, Oxford University Press.

BORBASI, S., Y. CHAPMAN et K. WOTTON (2002). A Work in Progress: Nurses' Perceptions on Caring for End-of-Life Heart Failure Patients in Community and Hospital Settings, Adelaide, Flinders University.

BOWLING, A. (1995). Measuring Disease, Buckingham, Open University Press.

BUTOW, P.N., S. DOWSETT, R. HAGERTY et M. TATTERSALL (2002). "Communicating prognosis to patients with metastatic disease: What do they really want to know? ", Supportive Care Cancer, $\mathrm{n}^{\circ}$ 10, p. 161-168.

CAMPBELL, M.L. (1996). "Program assessment through outcomes analysis: Efficacy of a comprehensive supportive care team for endof-life-care », American Association of Critical Care Nurses, Clinical Issues, n 7, p. 159-167.

DAVIES, N. et M. CURTIS (2000). « Providing palliative care in end-stage heart failure », Professional Nurse, $\mathrm{n}^{\circ} 15$, p. 389-392.

DEATON, C. et K.L. GRADY (2004). «State of the science for cardiovascular nursing outcomes - Heart failure », Journal of Cardiovascular Nursing, $\mathrm{n}^{\circ} 19$, p. 329-338.

DÉCARIE, S. (2005). Développement d'une approche intégrée de soins curatifs et palliatifs aux personnes atteintes d'insuffisance cardiaque en centre hospitalier, Rapport de stage de maîtrise inédit, Faculté des sciences infirmières, Université de Montréal.

DOYON, O. (2002). Soins infirmiers cardiovasculaires 1 et 11, document inédit, Université du Québec à Trois-Rivières.

DUCHARME, A. (1999). "La défaillance cardiaque: Le diagnostic au cabinet», Le médecin du Québec, n 34, p. 63-73. 
EDMONDS, P.M., J.M. STUTTAFORD, J. PENNY, A.M. LYNCH et J. CHAMBERLAIN (1998). "Do hospital palliative care teams improve symptom control? Use of a modified STAS as an evaluation tool», Palliative Medicine, $\mathrm{n}^{\mathrm{o}}$ 12, p. 345-351.

ELLERSHAW, J. et S. WILKINSON (2003). Care of the Dying: A Pathway to Excellence, Oxford, Oxford University Press.

FAISINGER, R., K. FASSBENGER, C. BRENNEIS, P. BROWN, T. BRAUN, C. NEUMANN, A. VIGANO, J. HATCHER et P. JACOBS (2003). Évaluation économique de deux programmes régionaux de soins palliatifs pour les cancéreux en phase terminale, Otttawa, Fondation canadienne de la recherche sur les services de santé.

FAULL, C. et R. WOOF (2002). Palliative Care: An Oxford Core Text, Oxford, Oxford University Press.

FERRIS, F.D., H.M. BALFOUR, K. BOWEN, J. FARLEY, M. HARDWICK, C. LAMONTAGNE, M. LUNDY, A. SYME et P. WEST (2002). Modèle de guide des soins palliatifs: Fondé sur les principes et les normes de pratique nationaux, Ottawa, Association canadienne de soins palliatifs.

FINUCANE, T. E. (2002). "Care of patients nearing death: Another view», Journal of American Geriatric Society, n ${ }^{\circ}$ 50, p. 551-553.

GOODLIN, S.J., P.J. HAUPTMAN, R. ARNOLD, K. GRADY, R.E. HERSHBERGER, J. KUTNER, F. MASOUDI, J. SPERTUS, K. DRACUP, J.F. CLEARY, R. MEDAK, K. CRISPELL, P. ILEANA, B. STUART, C. WHITNEY, T. RECTOR, J. TENO, et D.G RENLUD (2004). "Consensus statement: Palliative and supportive care in advanced heart failure », Journal of Cardiac Failure, n ${ }^{\circ}$ 10, p. 200-204.

GREEN, L.W. et M.W. KREUTER (1999). Health Promotion Planning: An Educational and Environmental Approach, $3^{\text {e éd., }}$ Toronto, Mayfield.

HANRATTY, B., D. HIBBERT, F. MAIR, C. MAY, C. WARD, S. CAPEWELL, A. LITVA et G. CORCORAN (2002). "Doctors' perceptions of palliative care for heart failure: Focus group study », British Medical Journal, no 325 , p. 581-585.

HEARN, J. et I.J. HIGGINSON (1998). «Do specialist palliative care teams improve outcomes for cancer patients? A systematic literature review », Palliative Medicine, $\mathrm{n}^{\circ} 12$, p. 317-332.

HENDRICK, A. (2001). « Cost-effective outpatient management of persons with heart failure », Progress in Cardiovascular Nursing, $\mathrm{n}^{\circ} 16$, p. $50-56$.

HIGGINSON, I.J., I. FINLAY, D.M. GOODWIN, A.M. COOK, K. HOOD, A.G.K. EDWARDS, H.-R. DOUGLAS et C.E. NORMAN (2002). "Do hospital-based palliative teams improve care for patients or families at the end of life? », Journal of Pain and Symptom Management, $\mathrm{n}^{\circ}$ 23, p. 96-106.

HORNE, G. et S. PAYNE (2004). «Removing the boundaries: Palliative care for patients with heart failure», Palliative Medicine, no 18 , p. 291-296.
HUGHES, S.L., J. CUMMINGS, F. WEAVER, L. MANHEIM, B. BRAUN et K. CONRAD (1992). "A randomized trial of the cost effectiveness of VA hospital-based home care for the terminally ill », Health Services Research, $\mathrm{n}^{\circ} 26$, p. 801-817.

JUENGER, J., D. SCHELLBERG et S. KRAEMER (2002). « Health related quality of life in patients with congestive heart failure : Comparison with other chronic diseases and relation to functional variables ", Heart, $\mathrm{n}^{\circ} 87$, p. 235-241.

KRUMHOLZ, H.M., R.S. PHILLIPS, M.B. HAMEL, J.M. TENO, P. BELLAMY, S.K. BROSTE, R.M. CALIFF, H. VIDAILLET, R.B. DAVIS, L.H. MUHLBAIER, A.F. Jr. CONNORS, J. LYNN et L. GOLDMAN (1998). "Resuscitation preferences among patients with severe congestive heart failure: results from the SUPPORT project. Study to understand prognoses and preferences for outcomes and risks of treatments ", Circulation, $\mathrm{n}^{\circ} 98$, p. 648-655.

KUTNER, J.S., J.E. KASSNER et D.E. NOWELS (2001). «Symptom burden at the end of life: Hospice providers' perceptions », Journal of Pain and Symptom Management, $\mathrm{n}^{\circ} 21$, p. 473-480.

LAMBERT, P. et H. LECOMPTE (2000). Le citoyen: une personne du début... à la fin. Rapport sur l'État de la situation des soins palliatifs au Québec, Québec, Ministère de la Santé et des Services sociaux.

LEVENSON, J.W., E.P. McCARTY, J. LYNN, R.B. DAVIS et R.S. PHILLIPS (2000). «The last six months of life for patients with congestive heart failure ", Journal of American Geriatric Society, no 48, p. S101-S109.

LYNN, J., J. L. SCHUSTER et A. KABCENELL (2000). Improving Care for the End of Life, Oxford, Oxford University Press.

LYNN, J., J. TENO et P. RUSSELL (1997). "Perceptions by the family of the dying experience of older and seriously ill patients ", Annals of Internal Medicine, $\mathrm{n}^{\circ} 126$, p. 97-106.

MCCARTHY, M., J. ADDINGTON-HALL et J. LAY (1997). "Communication and choice in dying from heart disease ", Journal of the Royal Society of Medicine, $\mathrm{n}^{\circ}$ 90, p. 138-131.

MCCARTHY, M., M. LAY et J. ADDINGTON-HALL (1996). «Dying from heart disease ", Journal of the Royal College of Physicians of London, $\mathrm{n}^{\circ}$ 30, p. 325-328.

MCMURRAY, J.J. et S. STEWART (2000). "Epidemiology, aetiology and prognosis of heart failure», Heart, no 83, p. 596-602.

MEIER, D.E. (2002). "Overview of cancer pain and palliative care », Journal of Pain and Symptom Management, $\mathrm{n}^{\circ}$ 24, p. 97-106.

MINISTÈRE DE LA SANTÉ ET DES SERVICES SOCIAUX (2004). Politique en soins palliatifs de fin de vie, Québec, Ministère de la Santé et des Services sociaux.

MINISTÈRE DE LA SANTÉ ET DES SERVICES SOCIAUX (1998). Registre des événements démographiques du Québec, fichier des décès, Unité d'études et d'analyses, Québec, Ministère de la Santé et des Services sociaux.
MOSER, D.K. et P.I. WORSTER (2000). "Effect of psychosocial factors on physiologic outcomes in patients with heart failure", Journal of Cardiovascular Nursing, $\mathrm{n}^{\circ} 14$, p. 106-115.

MURRAY, S.A., K. BOYD, M. KENDALL, A. WORTH, T.F. BENTON et H. CLAUSEN (2002). « Dying of lung cancer or cardiac failure: Prospective qualitative interview study of patients and their carers in the community ", British Medical Journal, no 325, p. 929-933.

MURRAY, S.A., M. KENDALL, K. BOYD, A. WORTH et T.F. BENTON (2004). « Exploring the spiritual needs of peoples dying of lung cancer or heart failure: A prospective qualitative interview study of patients and their careers », Palliative Medicine, $\mathrm{n}^{\circ} 18$, p. 39-45.

O'BRIEN, T., J. WELSH et F.G. DUNN (1998). «ABC of palliative care: Non-malignant conditions », British Medical Journal, $\mathrm{n}^{\circ} 16$, p. 286-289.

PANTILAT, S.Z. et A.E. STEIMLE (2004). "Palliative care for patients with heart failure ", Journal of American Medical Association, $\mathrm{n}^{\circ} 26$, p. 2476-2482.

RÉGIE RÉGIONALE DE LA SANTÉ ET DES SERVICES SOCIAUX DE MONTRÉAL-CENTRE (1999). Les réseaux des services de santé de première ligne: le cas des services dispensés aux personnes atteintes d'insuffisance cardiaque dans le Sud-Ouest de Montréal: rapport descriptif, Montréal, Régie régionale de la santé et des services sociaux de Montréal-Centre, Direction de la santé publique.

REMME, W.J. et K. SWEDBERG (2001). "Guidelines for the diagnosis and treatment of chronic heart failure », European Heart Journal, $\mathrm{n}^{\circ} 22$, p. 1527-1560.

RILEY, J. et L. BLUE (2001). «Assessing and managing chronic heart failure», Professional Nurse, $\mathrm{n}^{\circ}$ 16, p. 1112-1115.

SAUNDERS, C. (1999). «Pain and impending pain ", dans P. WALL et R. MELZACK (dir.), Textbook of Pain, $2^{\mathrm{e}}$ édition, Londres, Churchill Livingstone.

SINGER, P.A., D.K. MARTIN et M. KELNER (1999). "Quality end-of-life care patient's perspectives ", Journal of American Medical Association, $\mathrm{n}^{\circ}$ 281, p. 163-168.

STEINHAUSER, K., N.A. CHRISTAKIS, E.C. CLIPP, M. McNEILLY, S. GRAMBOW, J. PARKER et J.A. TULSKY (2001). " Preparing for the end of life: Preferences of patients, families, physicians, and other care providers ", Journal of Pain and Symptom Management, $\mathrm{n}^{\circ} 22$, p. 727-737.

VINEY, L.L., B.M. WALKER, T. ROBERTSON, B. LILLEY et C. EWAN (1994). «Dying in palliative care units and in hospital: A comparison of the quality of life of terminal cancer patients », Journal of Consulting Clinical Psychology, $\mathrm{n}^{\circ}$ 62, p. 157-164.

WOTTON, K., S. BORBASI et M. REDDEN (2005). «When else has failed: Nurses' perception of factors influencing palliative care for patients with end-stage heart failure», Journal of Cardiovascular Nursing, $\mathrm{n}^{\circ} 20$, p. $18-25$. 